
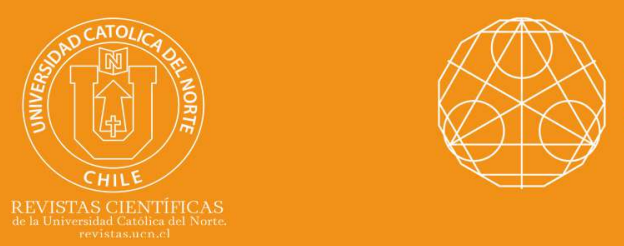

\title{
Inverse eigenproblems of real symmetric doubly arrowhead matrices
}

H. Pickmann-Soto ${ }^{1}$ (1) orcid.org/0000-0002-2472-0550

Susana Arela ${ }^{2}$ 이 orcid.org/0000-0003-4214-6908

J. Egaña ${ }^{3}$ (1) orcid.org/0000-0003-0033-2120

D. Carrasco Olivera ${ }^{4}$ () orcid.org/0000-0003-2868-534X

Universidad de Tarapacá, Dept. de Matemática, Arica, Chile.

1 hpickmanns@academicos.uta.cl; ${ }^{2}$ susana.arela.p@gmail.com

${ }^{3}$ Universidad Católica del Norte, Dept. de Matemáticas, Antofagasta,Chile.

■ jegana@ucn.cl

${ }^{4}$ Universidad del Bío-Bío, Dept. de Matemáticas, GISDA. Concepción, Chile.

—dcarrasc@ubiobio.cl

Received: February 2020 | Accepted: April 2020

\section{Abstract:}

We consider two inverse eigenproblems for a real symmetric doubly arrowhead matrix $A_{n}^{(())}$, which consist of constructing $A_{n}^{(())}$from two special kinds of spectra information. These problems were introduced in [11], where the principal results are sufficient conditions for both problems to have a real solution. In this paper, we improve such conditions, in the sense that one of the given conditions implies the rest. The results are constructive and generate one numerical procedure to construct the solution matrix $A_{n}^{\left({ }^{(q)}\right.}$.

Keywords: Real Symmetric matrices; Doubly arrowhead matrices; Inverse eigenvalue problem.

MSC (2020): 15A18; 15A42; 65F18.

\section{Cite this article as (IEEE citation style):}

H. Pickmann, S. Arela, J. Egaña, and D. Carrasco, "Inverse eigenproblems of real symmetric doubly arrowhead matrices", Proyecciones (Antofagasta, On line), vol. 39, no. 5, pp. 1315-1331, Oct. 2020, doi: 10.22199/issn.0717-6279-2020-05-0080.

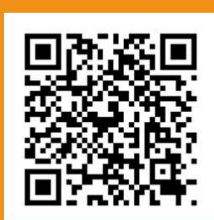

Article copyright: (C) 2020 H. Pickmann, S. Arela, J. Egaña, and D. Carrasco. This is an open access article distributed under the terms of the Creative Commons Licence, which permits unrestricted use and distribution provided the original author and source are credited. 


\section{Introduction}

This work is motived by two inverse eigenproblems, introduced in [11], for an $n \times n$ real symmetric doubly arrowhead matrix $A_{n}^{(q)}$ of the form:

$$
A_{n}^{(q)}=\left(\begin{array}{ccccccc}
a_{1} & & & b_{1} & & & \\
& \ddots & & \vdots & & & \\
& & a_{q-1} & b_{q-1} & & & \\
b_{1} & \cdots & b_{q-1} & a_{q} & b_{q} & \cdots & b_{n-1} \\
& & & b_{q} & a_{q+1} & & \\
& & & \vdots & & \ddots & \\
& & & b_{n-1} & & & a_{n}
\end{array}\right), a_{j} \in \mathbf{R}, b_{j}>0
$$

where $q$ can take some value between 1 and $n$. The matrix $A_{n}^{(q)}$ is a generalization of a real symmetric arrowhead matrix, in the sense that, if $q=1$ or $q=n$ we have a upward or downward arrowhead matrix, respectively (see [10], [13]). The form of a matrix $A_{n}^{(q)}$ is particularly special; this is obtained by a permutation of the row (or column) 1 with the row (or column) $q$ of a symmetric upward arrowhead matrix. On the other hand, it coincides with the structure of the inverse of a downward arrowhead matrix, whose element in the position $(q, q)(1<q<n)$, is equal to zero (see [7]). An analogous situation happens, in both cases, if we have a downward or upward arrowhead matrix, respectively.

The problem of constructing a symmetric doubly arrowhead matrix from certain spectral information is important in many applications, such as vibration theory, structural design, control theory, etc. In particular, for $q=1$ or $q=n$, these matrices are associated with a discrete dynamic model of a mechanical structure. Besides, the symmetric doubly arrowhead matrices have other important applications in the theory of graphs. Indeed, the matrix $A_{n}^{(q)}$ has an associated star graph of $n$ vertices, whose root of the star (vertex to which all the others are connected) corresponds to the $q$-th vertex, that is, to the entries of the $q$-th row and $q$-th column of the matrix $A_{n}^{(q)}$. Such matrices are called acyclic since they have an associated star graph, which is a type of tree (graph connected without cycles).

The study of graph theory has been of great importance for various sciences, such as chemistry, physics, operations research, combinatorial optimization, and computer science. In particular, among the applications of the star graph, we have the star network, a computer network modeled 
after the star graph that is important in distributed computing, a field of computer science that studies distributed systems.

In the context of the inverse eigenproblem, the classic spectral data known to construct a symmetric matrix are all the eigenvalues of the matrix and all the eigenvalues of a leading principal submatrix of order $n-1$ (see [1]-[3], [5]-[6]). However, from the practical point of view, only some eigenvalues and/or eigenvectors of the matrix or submatrices can be known (see [2], [6]). In this sense, in [12], the authors consider a special kind of spectral data, this is, the minimal and maximal eigenvalues of all leading principal submatrices of $A_{n}^{(q)}$ or an eigenvalue of each leading principal submatrix of $A_{n}^{(q)}$, together with an eigenpair of it. Initially, this special kind of spectral data was introduced by Peng et al. in [9], and subsequently, it has been considered by several authors (see [4], [8], [10]-[13]).

Throughout this paper, we shall denote as $I_{j}$ the $j \times j$ identity matrix, $A_{j}^{(q)}$ the $j \times j$ leading principal submatrix of $A_{n}^{(q)}, P_{j}^{(q)}(\lambda)$ the characteristic polynomial of $A_{j}^{(q)}, \lambda_{1}^{(j, q)} \leq \lambda_{2}^{(j, q)} \leq \cdots \leq \lambda_{j}^{(j, q)}$ the eigenvalues of $A_{j}^{(q)}$, and $\sigma\left(A_{j}^{(q)}\right)$ the spectrum of $A_{j}^{(q)}$. In the case where we consider only one eigenvalue of $A_{j}^{(q)}$, it will be denoted by $\lambda^{(j, q)}$, and by $\mathbf{x}^{(q)}$ its respective eigenvector.

In this work, we will discuss the inverse problems considered in [11], which are formulated as follows:

Problem 1. : Given the real numbers $\lambda_{1}^{(j, q)}$ and $\lambda_{j}^{(j, q)}, j=1,2, \ldots, n$, find necessary and sufficient conditions for the existence of an $n \times n$ matrix $A_{n}^{(q)}$ of the form (1.1), such that $\lambda_{1}^{(j, q)}$ and $\lambda_{j}^{(j, q)}$ are respectively, the minimal and maximal eigenvalues of the $j \times j$ leading principal submatrix $A_{j}^{(q)}$ of $A_{n}^{(q)}, j=1,2, \ldots, n$.

Problem 2. : Given the real numbers $\lambda^{(j, q)}, j=1,2, \ldots, n$ and a real vector $\mathbf{x}^{(q)}=\left(x_{1}^{(q)}, \ldots, x_{n}^{(q)}\right)^{T}$, find necessary and sufficient conditions for the existence of an $n \times n$ matrix $A_{n}^{(q)}$ of the form (1.1), such that $\lambda^{(j, q)}$ is an eigenvalue of the $j \times j$ leading principal submatrix $A_{j}^{(q)}$ of $A_{n}^{(q)}, j=$ $1,2, \ldots, n-1$, and $\left(\lambda^{(n, q)}, \mathbf{x}^{(q)}\right)$ is an eigenpair of $A_{n}^{(q)}$.

Regarding to Problem 1, in [[11], Theorem 2.2] it is said that there is a solution if 


$$
\lambda_{1}^{(n, q)}<\cdots<\lambda_{1}^{(q-1, q)}=\cdots=\lambda_{1}^{(1, q)}<\lambda_{2}^{(2, q)}<\cdots<\lambda_{n}^{(n, q)}
$$

and there exist real solutions $a_{q}, b_{k}>0, k=1, \ldots, q-1$ of the system of equations

$$
P_{q}^{(q)}\left(\lambda_{j}^{(q, q)}\right)=\left(\lambda_{j}^{(q, q)}-a_{q}\right) P_{q-1}^{(q)}\left(\lambda_{j}^{(q, q)}\right)-\sum_{k=1}^{q-1} b_{k}^{2} \prod_{\substack{i=1 \\ i=k}}^{q-1}\left(\lambda_{j}^{(q, q)}-\lambda_{i}^{(i, q)}\right)=0,
$$

for $j=1, q$.

In the Theorem 1, Second Step, we prove that the system of equations given in condition (1.3) has a solution if condition (1.2) is satisfied.

On the other hand, the sufficient conditions to determine a solution to Problem 2 given in [[11], Theorem 3.1] are:

$$
\begin{gathered}
x_{k}^{(q)}=0, \quad k=1, \ldots, n, \\
P_{q-1}^{(q)}\left(\lambda^{(q, q)}\right)=0
\end{gathered}
$$

and there exists a real solution $b_{j-1}$ of the equation

$b_{j-1}^{2} \prod_{\substack{i=1 \\ i=q}}^{j-1}\left(\lambda^{(j, q)}-a_{i}\right)-b_{j-1} \frac{x_{q}^{(q)}}{x_{j}^{(q)}} P_{j-1}^{(q)}\left(\lambda^{(j, q)}\right)+\left(\lambda^{(n, q)}-\lambda^{(j, q)}\right) P_{j-1}^{(q)}\left(\lambda^{(j, q)}\right)=0$,

$j=q+1, \ldots, n-1$.

In this case, we prove in Theorem 2 that the condition (1.5) is satisfied if we consider the minimal eigenvalue of the leading principal submatrix $A_{q}^{(q)}$. Furthermore, with an adequate order of the given eigenvalues and the condition (1.4), we also prove that the quadratic equations given in (1.6) have positive discriminants.

The paper is organized as follows: In Section 2, through Theorems 1 and 2, we prove that the Theorems 2.2 and 3.1 in [11] can be substantially improved. Indeed, we guarantee this by providing a particular solution to Problems 1 and 2. In Section 3, we will give some numerical examples to analyze the accuracy and efficiency of the results. Our results are constructive, in the sense that they generate an algorithmic procedure to construct the solution matrices. 


\section{New Sufficient Conditions for Problems 1 and 2}

In this section, we give new sufficient conditions for Problems 1 and 2 to have a real solution. In each case, we include the algorithm that is derived from our results. First, we recall the following results:

Lemma 1. [11] Let $A_{n}^{(q)}$ be an $n \times n$ matrix of the form (1.1). Then the sequence of characteristic polynomials $\left\{P_{j}^{(q)}(\lambda)\right\}_{j, q=1}^{n}$ satisfies the recurrence relation:

$$
\begin{gathered}
P_{j}^{(q)}(\lambda)=\prod_{i=1}^{j}\left(\lambda-a_{i}\right) j=1, \ldots, q-1 . \\
P_{j}^{(q)}(\lambda)=\left(\lambda-a_{j}\right) P_{j-1}^{(q)}(\lambda)-j-1 k=1 \sum b_{k}^{2} \prod_{\substack{i=1 \\
i=k}}^{q-1}\left(\lambda-a_{i}\right) j=q . \\
P_{j}^{(q)}(\lambda)=\left(\lambda-a_{j}\right) P_{j-1}^{(q)}(\lambda)-b_{j-1}^{2} \prod_{\substack{i=1 \\
i=q}}^{j-1}\left(\lambda-a_{i}\right) j=q+1, \ldots, n,
\end{gathered}
$$

where $P_{0}^{(q)}(\lambda)=1$.

Lemma 2. [11] Let $P(\lambda)$ be a monic polynomial of degree $n$, with all real zeroes. If $\lambda_{1}$ and $\lambda_{n}$ are, respectively, the minimal and the maximal zero of $P(\lambda)$, then

1. If $\mu<\lambda_{1}$, we have that $(-1)^{n} P(\mu)>0$.

2. If $\mu>\lambda_{n}$, we have that $P(\mu)>0$.

Notice that for the sequence of polynomials of Lemma 1 , if $\mu<\lambda_{1}^{(j, q)}$, then $(-1)^{j} P_{j}^{(q)}(\mu)>0$ and if $\mu>\lambda_{j}^{(j, q)}$, then $P_{j}^{(q)}(\mu)>0$. The minimal and maximal eigenvalues of $A_{j}^{(q)}$ will be called extremal eigenvalues.

Lemma 3. (Cauchy Interlacing Theorem) Let $A_{n}^{(q)}$ be an $n \times n$ real symmetric matrix with eigenvalues $\lambda_{1}^{(n, q)} \leq \lambda_{2}^{(n, q)} \leq \cdots \leq \lambda_{n}^{(n, q)}$. Let $A_{r}^{(q)}$, with eigenvalues $\lambda_{1}^{(r, q)} \leq \lambda_{2}^{(r, q)} \leq \cdots \leq \lambda_{n-1}^{(r, q)}$, the principal submatrix of $A_{n}^{(q)}$, obtained by deleting the $r-$ th row and $r-t h$ column of $A_{n}^{(q)}$. Then $\lambda_{1}^{(n, q)} \leq \lambda_{1}^{(r, q)} \leq \lambda_{2}^{(n, q)} \leq \cdots \leq \lambda_{n-1}^{(n, q)} \leq \lambda_{n-1}^{(r, q)} \leq \lambda_{n}^{(n, q)}$. 
The following theorem is the main result of this paper related to Problem 1:

Theorem 1. Let the real numbers $\lambda_{1}^{(j, q)}$ and $\lambda_{j}^{(j, q)}, j, q=1,2, \ldots, n$, be given. If

$$
\lambda_{1}^{(n, q)}<\ldots<\lambda_{1}^{(q-1, q)}=\ldots=\lambda_{1}^{(1, q)}<\lambda_{2}^{(2, q)}<\ldots<\lambda_{n}^{(n, q)},
$$

with $\lambda_{1}^{(0, q)}=\lambda_{1}^{(1, q)}$, then there exists an $n \times n$ real symmetric doubly arrowhead matrix $A_{n}^{(q)}$ of the form (1.1), such that $\lambda_{1}^{(j, q)}$ and $\lambda_{j}^{(j, q)}$ are the extremal eigenvalues of the leading principal matrix $A_{j}^{(q)}, j=1,2, \ldots, n$.

Proof. Suppose that the real numbers $\lambda_{1}^{(j, q)}$ and $\lambda_{j}^{(j, q)}, j=1,2, \ldots, n$, satisfy condition (2.4). The proof will consist of three steps.

First Step: There exists a $j \times j$ matrix $A_{j}^{(q)}$ with extremal eigenvalues $\lambda_{1}^{(j, q)}$ and $\lambda_{j}^{(j, q)}$ for $j=1,2, \ldots, q-1$. Indeed, define the following matrix $A_{j}^{(q)}=$ $\operatorname{diag}\left\{\lambda_{1}^{(1, q)}, \lambda_{2}^{(2, q)}, \ldots, \lambda_{j}^{(j, q)}\right\}, j=1,2, \ldots, q-1$, with extremal eigenvalues $\lambda_{1}^{(j, q)}$ and $\lambda_{j}^{(j, q)}$.

Second Step: There exists a $q \times q$ matrix $A_{j}^{(q)}$ with extremal eigenvalues $\lambda_{1}^{(q, q)}$ and $\lambda_{q}^{(q, q)}$. Indeed, we will show that the system given by (1.3) has a solution if the condition (1.2) (or (2.4)) is satisfied. That is, we will show that the linear system of equations

$$
\begin{aligned}
& P_{q-1}^{(q)}\left(\lambda_{1}^{(q, q)}\right) a_{q}-\sum_{k=1}^{q-1} b_{k}^{2} \prod_{\substack{i=1 \\
i=k}}^{q-1}\left(\lambda_{1}^{(q, q)}-\lambda_{i}^{(i, q)}\right)-\lambda_{1}^{(q, q)} P_{q-1}^{(q)}\left(\lambda_{1}^{(q, q)}\right)=0 \\
& P_{q-1}^{(q)}\left(\lambda_{q}^{(q, q)}\right) a_{q}-\sum_{k=1}^{q-1} b_{k}^{2} \prod_{\substack{i=1 \\
i=k}}^{q-1}\left(\lambda_{q}^{(q, q)}-\lambda_{i}^{(i, q)}\right)-\lambda_{q}^{(q, q)} P_{q-1}^{(q)}\left(\lambda_{q}^{(q, q)}\right)=0
\end{aligned}
$$

has a solution $\left(a_{q}, b_{1}, b_{2}, \ldots, b_{q-1}\right)^{T} \in \mathbf{R}^{q}$. In fact, considering the condition (2.4) and Lemma 2 , we have that $P_{q-1}^{(q)}\left(\lambda_{1}^{(q, q)}\right)=0$ and $P_{q-1}^{(q)}\left(\lambda_{q}^{(q, q)}\right)=0$. Then, from equations (2.5) and (2.6) we obtain 


$$
\begin{aligned}
& a_{q}=\lambda_{1}^{(q, q)}-\frac{1}{P_{q-1}^{(q)}\left(\lambda_{1}^{(q, q)}\right)} \sum_{k=1}^{q-1} b_{k}^{2} \prod_{\substack{i=1 \\
i=k}}^{q-1}\left(\lambda_{1}^{(q, q)}-\lambda_{i}^{(i, q)}\right) \\
& a_{q}=\lambda_{q}^{(q, q)}-\frac{1}{P_{q-1}^{(q)}\left(\lambda_{q}^{(q, q)}\right)} \sum_{k=1}^{q-1} b_{k}^{2} \prod_{\substack{i=1 \\
i=k}}^{q-1}\left(\lambda_{q}^{(q, q)}-\lambda_{i}^{(i, q)}\right) .
\end{aligned}
$$

Now, as $\lambda_{q}^{(q, q)}-\lambda_{1}^{(q, q)}>0$, we have

$\sum_{\mathrm{k}=1}^{\mathrm{q}-1} \mathrm{~b}_{k}^{2}\left[\frac{1}{\left(\lambda_{q}^{(q, q)}-\lambda_{1}^{(q, q)}\right)}\left(\frac{1}{P_{q-1}^{(q)}\left(\lambda_{q}^{(q, q)}\right)} \prod_{\substack{i=1 \\ i=k}}^{q-1}\left(\lambda_{q}^{(q, q)}-\lambda_{i}^{(i, q)}\right)-\frac{1}{P_{q-1}^{(q)}\left(\lambda_{1}^{(q, q)}\right)} \prod_{\substack{i=1 \\ i=k}}^{q-1}\left(\lambda_{1}^{(q, q)}-\lambda_{i}^{(i, q)}\right)\right)\right]=1$.

Also, from the Lemma (2) and the condition (2.4), we obtain, for each $k=1,2, \ldots, q-1$,

$$
\frac{1}{P_{q-1}^{(q)}\left(\lambda_{q}^{(q, q)}\right)} \prod_{\substack{i=1 \\ i=k}}^{q-1}\left(\lambda_{q}^{(q, q)}-\lambda_{i}^{(i, q)}\right)>0
$$

and

$$
\frac{1}{P_{q-1}^{(q)}\left(\lambda_{1}^{(q, q)}\right)} \prod_{\substack{i=1 \\ i=k}}^{q-1}\left(\lambda_{1}^{(q, q)}-\lambda_{i}^{(i, q)}\right)<0
$$

Therefore, the equation (2.9) can be written as

$$
\sum_{k=1}^{q-1} B_{k}^{2}=1
$$

with

$$
B_{k}=\mathrm{b}_{k} \sqrt{\frac{1}{\left(\lambda_{q}^{(q, q)}-\lambda_{1}^{(q, q)}\right)}\left(\frac{1}{P_{q-1}^{(q)}\left(\lambda_{q}^{(q, q)}\right)} \prod_{\substack{i=1 \\ i=k}}^{q-1}\left(\lambda_{q}^{(q, q)}-\lambda_{i}^{(i, q)}\right)-\frac{1}{P_{q-1}^{(q)}\left(\lambda_{1}^{(q, q)}\right)} \prod_{\substack{i=1 \\ i=k}}^{q-1}\left(\lambda_{1}^{(q, q)}-\lambda_{i}^{(i, q)}\right)\right)},
$$


where the radicands of each root are positive.

Clearly, from the equations (2.11) and (2.7) (or (??)), we obtain infinite solutions for $b_{k}, k=1,2, \ldots, q-1$ and $a_{q}$. This proves the existence of a $q \times q$ matrix $A_{q}^{(q)}$. In particular, one solution can be obtained if we consider

$$
B_{1}=B_{2}=\cdots=B_{q-1}=\frac{1}{\sqrt{q-1}}, \quad q>1 .
$$

Thus,

$$
b_{k}=\frac{1}{\sqrt{\frac{q-1}{\left(\lambda_{q}^{(q, q)}-\lambda_{1}^{(q, q)}\right)}\left(\frac{1}{P_{q-1}^{(q)}\left(\lambda_{q}^{(q, q)}\right)} \prod_{\substack{i=1 \\ i=k}}^{q-1}\left(\lambda_{q}^{(q, q)}-\lambda_{i}^{(i, q)}\right)-\frac{1}{P_{q-1}^{(q)}\left(\lambda_{1}^{(q, q)}\right)} \prod_{\substack{i=1 \\ i=k}}^{q-1}\left(\lambda_{1}^{(q, q)}-\lambda_{i}^{(i, q)}\right)\right)}}
$$

and $a_{q}$ is computed by (2.7) (or (2.8)).

Third Step: There exists a $j \times j$ matrix $A_{j}^{(q)}, j=q+1, \ldots, n$, with the required spectral properties. Indeed, we proceed as in [[11], Theorem 2.2], that is, for $j=q+1, \ldots, n$

$$
a_{j}=\frac{\lambda_{1}^{(j, q)} P_{j-1}^{(q)}\left(\lambda_{1}^{(j, q)}\right) \prod_{\substack{i=1 \\ i=q}}^{j-1}\left(\lambda_{j}^{(j, q)}-a_{i}\right)-\lambda_{j}^{(j, q)} P_{j-1}^{(q)}\left(\lambda_{j}^{(j, q)}\right) \prod_{\substack{i=1 \\ i=q}}^{j-1}\left(\lambda_{1}^{(j, q)}-a_{i}\right)}{h_{j}}
$$

and

$$
b_{j-1}^{2}=\frac{\left(\lambda_{j}^{(j, q)}-\lambda_{1}^{(j, q)}\right) P_{j-1}^{(q)}\left(\lambda_{1}^{(j, q)}\right) P_{j-1}^{(q)}\left(\lambda_{j}^{(j, q)}\right)}{h_{j}}>0
$$

where

$$
\mathrm{h}_{j}=P_{j-1}^{(q)}\left(\lambda_{1}^{(j, q)}\right) \prod_{\substack{i=1 \\ i=q}}^{j-1}\left(\lambda_{j}^{(j, q)}-a_{i}\right)-P_{j-1}^{(q)}\left(\lambda_{j}^{(j, q)}\right) \prod_{\substack{i=1 \\ i=q}}^{j-1}\left(\lambda_{1}^{(j, q)}-a_{i}\right) \text {. This }
$$

completes the proof. 
Algorithm 1: Reconstruction of a doubly arrowhead symmetric matrix from extremal eigenvalues.

INPUT: The real numbers $\left\{\lambda_{1}^{(j, q)}, \lambda_{j}^{(j, q)}\right\}_{j=1}^{n}$, that satisfy the condition (2.4), and subindex $q$.

OUTPUT: An $n \times n$ matrix $A_{n}^{q}$ of the form (1.1) such that $\lambda_{1}^{(j, q)}$ and $\lambda_{j}^{(j, q)}$ are the minimal and the maximal eigenvalues of the leading principal submatrix $A_{j}^{q}, \quad j=1,2, \ldots, n$ of $A_{n}^{q}$.

1. for $j=1,2, \ldots, q-1$ do

2. $\quad$ set $a_{j}=\lambda_{j}^{(j, q)}$

3. end

4. for $j=1,2, \ldots, q-1$ do

5 . compute $b_{j}$ defined by $(2.12)$

6. end

7. for $j=q+1, q+2, \ldots, n$ do

8. $\quad$ compute $a_{j}$ defined by (2.13)

9. compute $b_{j-1}$ defined by the square root of (2.14)

10. end

Now we propose a better solution to Problem 2 through the following theorem:

Theorem 2. Let the set real numbers $\left\{\lambda_{1}^{(j, q)}\right\}_{j=1}^{n-1} \cup\left\{\lambda_{n}^{(n, q)}\right\}$ and a real vector $\mathbf{x}^{(q)}=\left(x_{1}^{(q)}, \ldots, x_{n}^{(q)}\right)^{T}$ be given. If

(2.15) $\lambda_{1}^{(n-1, q)}<\lambda_{1}^{(n-2, q)}<\ldots<\lambda_{1}^{(q, q)}<\lambda_{1}^{(q-1, q)} \leq \ldots \leq \lambda_{1}^{(1, q)}<\lambda_{n}^{(n, q)}$, with $\lambda_{1}^{(0, q)}=\lambda_{1}^{(1, q)}$, and

$$
x_{j}^{(q)}=0, \quad j=1, \ldots, n,
$$

then there exists an $n \times n$ matrix $A_{n}^{(q)}$ of the form (1.1), such that $\lambda_{1}^{(j, q)}$ is the minimal eigenvalue of its leading principal submatrix $A_{j}^{(q)}, j=1, \ldots, n-1$ and $\left(\lambda_{n}^{(n, q)}, x^{(q)}\right)$ is the maximal eigenpair of $A_{n}^{(q)}$. 
Proof. Suppose that the set real numbers $\left\{\lambda_{1}^{(j, q)}\right\}_{j=1}^{n-1} \cup\left\{\lambda_{n}^{(n, q)}\right\}$ and real vector $\mathbf{x}^{(q)}=\left(x_{1}^{(q)}, \ldots, x_{n}^{(q)}\right)^{T}$, satisfy the conditions (2.15) and (2.16), respectively. Then, by [[11], Theorem 3.1], from Lemmas 1 and 2, and $A_{n}^{(q)} \mathbf{x}^{(q)}=\lambda_{n}^{(n, q)} \mathbf{x}^{(q)}$, diagonal entries $a_{j}$ of the matrix $A_{n}^{(q)}$ can be computed by

$$
\begin{gathered}
a_{j}=\lambda_{1}^{(j, q)}, \quad j=1, \ldots, q-1, \\
a_{q}=\frac{\lambda_{1}^{(q, q)} P_{q-1}^{(q)}\left(\lambda^{(q, q)}\right)--\sum_{k=1}^{q-1} b_{k}^{2} \prod_{\substack{i=1 \\
i=k}}^{q-1}\left(\lambda_{1}^{(q, q)}-a_{i}\right)}{P_{q-1}^{(q)}\left(\lambda_{1}^{(q, q)}\right)},
\end{gathered}
$$

and

$$
a_{j}=\lambda_{n}^{(n, q)}-b_{j-1} \frac{x_{q}^{(q)}}{x_{j}^{(q)}}, \quad j=q+1, \ldots, n,
$$

and the entries $b_{j}$ by

$$
b_{j}=\left(\lambda_{n}^{(n, q)}-a_{j}\right) \frac{x_{j}^{(q)}}{x_{q}^{(q)}}, \quad j=1, \ldots, q-1 .
$$

Therefore, the matrix $A_{n}^{(q)}$ will be completely obtained if the entries $b_{j}, j=q, \ldots, n-1$ are computed. In this sense, we will prove that the quadratic equations that are the sufficient condition (1.6) of Theorem 3.1 in [11] always have at least one real solution. We will prove that discriminants

$$
\Delta_{j}=\left[\frac{x_{q}^{(q)}}{x_{j}^{(q)}} P_{j-1}^{(q)}\left(\lambda_{1}^{(j, q)}\right)\right]^{2}-4 \prod_{\substack{i=1 \\ i=q}}^{j-1}\left(\lambda_{1}^{(j, q)}-a_{i}\right)\left(\lambda_{n}^{(n, q)}-\lambda_{1}^{(j, q)}\right) P_{j-1}^{(q)}\left(\lambda_{1}^{(j, q)}\right),
$$

$j=q+1, \ldots, n-1$ are positive. In fact, is known that

$$
\lambda_{1}^{(j, q)} \leq a_{i} \leq \lambda_{j}^{(j, q)}, \quad j=1,2, \ldots, n-1, \quad i=1,2, \ldots, j .
$$

In particular, for $j=q$ and (2.15), we have

$$
\lambda_{1}^{(q+1, q)}<\lambda_{1}^{(q, q)} \leq a_{i} \leq \lambda_{j}^{(q, q)}, \quad i=1,2, \ldots, q .
$$


Now, the second term of the expression of the right side of $\Delta_{j}$ can be written as follows:

$$
(-1) 4 \prod_{\substack{i=1 \\ i=q}}^{j-1}\left(a_{i}-\lambda_{1}^{(j, q)}\right)\left(\lambda_{n}^{(n, q)}-\lambda_{1}^{(j, q)}\right)(-1)^{j-1} P_{j-1}^{(q)}\left(\lambda_{1}^{(j, q)}\right)
$$

where, $\left(a_{i}-\lambda_{1}^{(j, q)}\right)>0,\left(\lambda_{n}^{(n, q)}-\lambda_{1}^{(j, q)}\right)>0$ and $(-1)^{j-1} P_{j-1}^{(q)}\left(\lambda_{1}^{(j, q)}\right)>$ 0 , by Lemmas 2 and 3 . Therefore, all the discriminants $\Delta_{j}$ are positive, i.e., the entries $b_{j-1}, j=q+1, \ldots, n$ can be computed as

$$
b_{j-1}=\frac{\frac{x_{q}^{(q)}}{x_{j}^{(q)}} \mathrm{P}_{j-1}^{(q)}\left(\lambda_{1}^{(j, q)}\right) \pm \sqrt{\left[\frac{x_{q}^{(q)}}{x_{j}^{(q)}} \mathrm{P}_{j-1}^{(q)}\left(\lambda_{1}^{(j, q)}\right)\right]^{2}-4 \prod_{\substack{i=1 \\ i=q}}^{j-1}\left(\lambda_{1}^{(j, q)}-\mathrm{a}_{i}\right)\left(\lambda_{n}^{(n, q)}-\lambda_{1}^{(j, q)}\right) \mathrm{P}_{j-1}^{(q)}\left(\lambda_{1}^{(j, q)}\right)}}{2_{\substack{i=1 \\ i=q}}^{j-1}\left(\lambda_{1}^{(j, q)}-\mathrm{a}_{i}\right)} .
$$

This completes the proof.

Algorithm 2: Reconstruction of a doubly arrowhead symmetric matrix from minimal eigenvalues and eigenpair.

INPUT: The real numbers $\left\{\lambda_{1}^{(j, q)}\right\}_{j=1}^{n} \cup\left\{\lambda_{n}^{(n, q)}\right\}$ and the vector $x^{(q)}=\left(x_{1}^{q}, x_{2}^{q}, \ldots, x_{n}^{q}\right)$, that satisfy the conditions (2.15) and (2.16), respectively, and subindex $q$.

OUTPUT: An $n \times n$ matrix $A_{n}^{q}$ of the form (1.1) such that $\lambda_{1}^{(j, q)}$ is the minimal eigenvalue of the leading principal submatrix $A_{j}^{q}, \quad j=1,2, \ldots, n$ of $A_{n}^{q}$ and $\left(\lambda_{n}^{(n, q)}, x^{(q)}\right)$ is the eigenpair of $A_{n}^{q}$, with being $\lambda_{n}^{(n, q)}$ the maximal eigenvalue of $A_{n}^{q}$.

1. for $j=1,2, \ldots, q-1$ do

2. $\quad$ set $a_{j}=\lambda_{1}^{(j, q)}$

3. end

4. compute $a_{q}$ defined by (2.17)

5. for $j=q+1, q+2, \ldots, n$ do

6. compute $a_{j}$ defined by (2.18) 
7. end

8. for $j=1,2, \ldots, q-1$ do

$9 . \quad$ compute $\quad b_{j}$ defined by $(2.19)$

10. end

11. for $j=q+1, q+2, \ldots, n$ do

12. compute $b_{j-1}$ defined by $(2.20)$

13. end

\section{Numerical examples}

In this section, we present the results and performance of the Algorithms 1 and 2 in determining a solution of Problems 1 and 2, respectively. Extensive experiments have been realized. All experiments were done in Matlab R2019a on an Intel CORE i7 8-th gen processor, DDR4 2133MHz SDRAM Onboard Memory, up to 16 GB SDRAM with IEEE doubly precision arithmetic.

In order to show the matrix $A_{n}^{(q)}$ that Algorithms 1 and 2 reconstructs, in Example 1 and 2, we execute experiments from an initial spectral data given, satisfying the conditions of Theorems 1 and 2 .

Example 1. In Example 1, two test are done showing the matrix $A_{n}^{(q)}$ recovered, whose initial data are evenly distributed. In the first test, we set $n=7$ and $q=3$, and the initial real numbers $\lambda_{1}^{(j, 3)}$ and $\lambda_{j}^{(j, 3)}, j=1,2, \ldots, 7$ in the second and third row of Table 1 are listed.

\begin{tabular}{|c|c|c|c|c|}
\hline$\lambda_{1}^{(j, 3)}$ & $\lambda_{j}^{(j, 3)}$ & $j$ & $a_{i}^{(7,3)}$ & $b_{i}^{(7,3)}$ \\
\hline 1.0000 & & 1 & 1.0000 & 3.1623 \\
\hline 1.0000 & 4.0000 & 2 & 4.0000 & 2.6458 \\
\hline-3.0000 & 6.0000 & 3 & 0,5000 & 4.0620 \\
\hline-5.0000 & 7.0000 & 4 & 4.0000 & 3.7000 \\
\hline-6.0000 & 8.0000 & 5 & 1.6338 & 5.1235 \\
\hline-9.0000 & 9.0000 & 6 & -3.6633 & 11.5640 \\
\hline-14.0000 & 15.0000 & 7 & 1.0535 & \\
\hline
\end{tabular}

Once we apply Algorithm 1, the entries of the matrix $A_{7}^{(3)}$ reconstructed in the second and third row are listed in Table 2. To verify that the matrix 
obtained is correct, we calculated the spectra $\sigma\left(A_{j}^{(3)}\right)$ of each submatrix $A_{j}^{(3)}, j=1,2, \ldots, 7$, which are listed in Table 3. Here, the bold numbers correspond to the minimal and maximal eigenvalues of $A_{j}^{(3)}$ recovered. We can see that these numbers coincide exactly with the initial data.

Table 3: The spectra $\sigma\left(A_{j}^{(3)}\right)$ of each submatrix $A_{j}^{(3)}$

\begin{tabular}{ccccccccc}
\hline$j$ & $\sigma\left(A_{j}^{(3)}\right)$ & & & & & & \\
\hline 1 & $\mathbf{1 . 0 0 0 0}$ & & & & & & \\
2 & $\mathbf{1 . 0 0 0 0}$ & $\mathbf{4 . 0 0 0 0}$ & & & & & \\
3 & $-\mathbf{3 . 0 0 0 0}$ & 2.5000 & $\mathbf{6 . 0 0 0 0}$ & & & & \\
4 & $\mathbf{- 5 . 0 0 0 0}$ & 0.7657 & 3.1343 & $\mathbf{7 . 0 0 0 0}$ & & & \\
5 & $\mathbf{- 6 . 0 0 0 0}$ & 0.7152 & 1.3282 & 3.4904 & $\mathbf{8 . 0 0 0 0}$ & & \\
6 & $-\mathbf{9 . 0 0 0 0}$ & -1.7916 & 0.7346 & 1.3480 & 3.5795 & $\mathbf{9 . 0 0 0 0}$ & \\
7 & $-\mathbf{1 4 . 0 0 0 0}$ & -3.0167 & 0.4651 & 1.0037 & 1.5855 & 3.8863 & $\mathbf{1 5 . 0 0 0 0}$
\end{tabular}

In the second test, we set $n=7$ and $q=4$, and the initial real numbers $\lambda_{1}^{(j, 4)}, j=1,2, \ldots 6$ and $\lambda_{7}^{(7,4)}$, and the components $\mathbf{x}_{j}^{(4)}, j=1,2, \ldots 7$ of the vector $\mathbf{x}^{(4)}$ in the second and third row of Table 4 are listed.

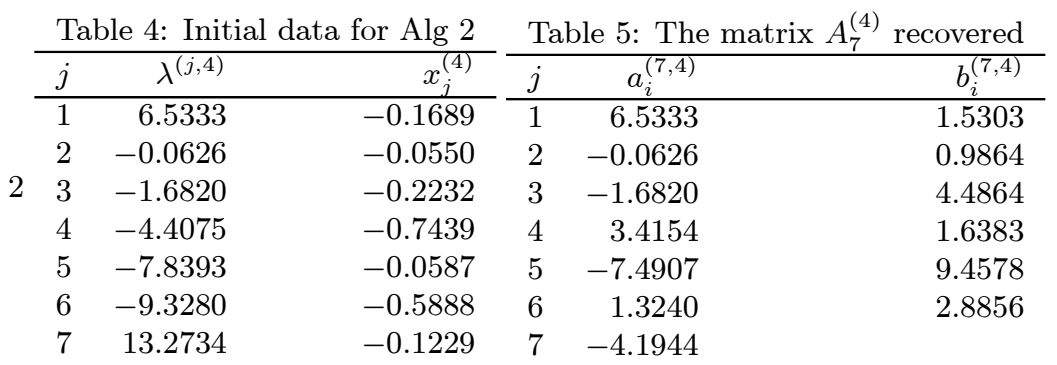

Table 5, shows the matrix $A_{7}^{(4)}$, reconstructed by Algorithm 2, and Table 6 shows the spectra $\sigma\left(A_{j}^{(4)}\right)$ of $A_{j}^{(4)}, \quad j=1,2, \ldots, 7$ and eigenpair $\left(\lambda^{(7,4)}, \mathbf{x}^{(4)}\right)$. Again, the results show that the reconstructed matrix $A_{7}^{(4)}$ is as expected.

Table 6: The spectra $\sigma\left(A_{j}^{(4)}\right)$ of each submatrix $A_{j}^{(4)}$

\begin{tabular}{|c|c|c|c|c|c|c|c|}
\hline$j$ & $\sigma\left(A_{j}^{(4)}\right)$ & & & & & & \\
\hline 1 & 6.5333 & & & & & & \\
\hline 2 & -0.0626 & 6.5333 & & & & & \\
\hline 3 & -1.6820 & -0.0626 & 6.5333 & & & & \\
\hline 4 & -4.4075 & -0.1230 & 5.0369 & 7.6978 & & & \\
\hline 5 & -7.8393 & -4.2035 & -0.1217 & 5.1193 & 7.7588 & & \\
\hline 6 & -9.3280 & -6.9174 & -1.0817 & -0.0430 & 6.3993 & 13.0082 & \\
\hline 7 & -9.8703 & -7.1057 & -3.7729 & -1.0441 & -0.0422 & 6.4049 & 13.2734 \\
\hline $13.2734 x^{(4)}$ & -2.2420 & -0.7304 & -2.9621 & -9.8743 & -0.7791 & -7.8154 & -1.6312 \\
\hline
\end{tabular}


Example 2. In this example, we show two extensive tests of dimensions $n=1,2, \ldots, 100$, where the initial spectral data are randomly distributed in such a way showing the accuracy and efficiency of Algorithms 1 and 2.

Randomly spectral data are indirectly generated, that is, with $2 \mathrm{n}-1$ random numbers generated by Matlab randn routine, we obtain a random matrix $\widehat{A}_{n}^{(q)}$. Then, for each $n=1,2, \ldots, 100$, we calculate the minimal and maximal eigenvalues $\widehat{\lambda}_{1}^{(j, q)}$ and $\hat{\lambda}_{j}^{(j, q)}$ of $\widehat{A}_{j}^{(q)}, j=1,2, \ldots, n$ and the eigenpair $\left(\widehat{\lambda}^{(n, q)}, \widehat{\mathbf{x}}^{(q)}\right)$ of $\widehat{A}_{n}^{(q)}$, using the Matlab eig routine, being this the initial spectral data of Algorithms 1 and 2 to calculate the matrix $A_{n}^{(q)}$.

Once the matrix $A_{n}^{(q)}$ is reconstructed, the accuracy of Algorithm 1 is measured by computing the relative error $e_{\lambda}$ defined by

$$
e_{\lambda_{1}}=\frac{\|\hat{\lambda}-\lambda\|_{\infty}}{\|\hat{\lambda}\|_{\infty}}
$$

where $\hat{\lambda}=\left(\widehat{\lambda}_{1}^{(n, q)}, \widehat{\lambda}_{1}^{(n-1, q)}, \ldots, \widehat{\lambda}_{1}^{(2, q)}, \widehat{\lambda}_{1}^{(1, q)}, \widehat{\lambda}_{2}^{(2, q)}, \ldots, \widehat{\lambda}_{n-1}^{(n-1, q)}, \widehat{\lambda}_{n}^{(n, q)}\right)^{T}$ is the vector of the random initial data and $\lambda=\left(\lambda_{1}^{(n, q)}, \lambda_{1}^{(n-1, q)}, \ldots, \lambda_{1}^{(2, q)}, \lambda_{1}^{(1, q)}, \lambda_{2}^{(2, q)}, \ldots, \lambda_{n-1}^{(n-1, q)}, \lambda_{n}^{(n, q)}\right)^{T}$ is the vector of the minimal and maximal eigenvalues of the matrix $A_{n}^{(q)}$. While for Algorithm 2, we define the relative errors

$$
e_{\lambda_{2}}=\frac{\|\tilde{\lambda}-\lambda\|_{\infty}}{\|\tilde{\lambda}\|_{\infty}} \text { and } e_{x}=\frac{\|\widehat{\mathbf{x}}-\mathbf{x}\|_{\infty}}{\|\widehat{\mathbf{x}}\|_{\infty}}
$$

$\widetilde{\lambda}=\left(\widetilde{\lambda}_{1}^{(n, q)}, \widetilde{\lambda}_{1}^{(n-1, q)}, \ldots, \widetilde{\lambda}_{1}^{(2, q)}, \widetilde{\lambda}_{1}^{(1, q)}\right)^{T}$ and $\widetilde{\mathbf{x}}=\left(\widetilde{\lambda}_{n}^{(n, q)}, \widetilde{x}_{1}^{q}, \widetilde{x}_{2}^{q}, \ldots, \widetilde{x}_{n}^{q}\right)^{T}$ are the vectors of the random initial data and $\lambda=\left(\lambda_{1}^{(n, q)}, \lambda_{1}^{(n-1, q)}, \ldots, \lambda_{1}^{(2, q)}, \lambda_{1}^{(1, q)}\right)^{T}$ and $\mathbf{x}=\left(\lambda_{n}^{(n, q)}, x_{1}^{q}, x_{2}^{q}, \ldots, x_{n}^{q}\right)^{T}$ are, respectively, the vectors of the minimal eigenvalues and eigenpair of the matrix $A_{n}^{(q)}$.

Table 7 shows the error $e_{\lambda_{1}}$ for Algorithm 1, while Table 8 shows the errors $e_{\lambda_{2}}$ and $e_{x}$ for Algorithm 2. Both Tables are organized as follows: the first column displays the dimension $n$. To display the error $e_{\lambda_{1}}$, there are three columns: the first one displays the average over the 1000 tests made; the second and third display, respectively, the smallest and largest value for the error among the 1000 experiments. Similarly, to show the errors $e_{\lambda_{2}}$ and $e_{x}$, the first columns of Table 8 show the average of the 1000 tests performed, 
while the second columns display the smallest value for the error among the 1000 tests and the third columns the largest value.

Table 7: Relative error $e_{\lambda_{1}}$ for Alg 1

\begin{tabular}{cccc}
\hline$n$ & \multicolumn{3}{c}{$e_{\lambda_{1}}$} \\
\hline & Mean & Min & Max \\
\hline 10 & $6.17 e-16$ & $1.22 e-16$ & $1.54 e-15$ \\
25 & $1.71 e-15$ & $2.73 e-16$ & $2.67 e-15$ \\
50 & $1.35 e-15$ & $3.36 e-16$ & $2.73 e-15$ \\
75 & $1.45 e-15$ & $3.31 e-16$ & $3.22 e-15$ \\
100 & $1.47 e-15$ & $3.55 e-16$ & $3.24 e-15$
\end{tabular}

Table 8: Relative errors $e_{\lambda_{1}}$ and $e_{x}$ for Alg 2

\begin{tabular}{ccccccc}
\hline$n$ & \multicolumn{5}{c}{$e_{\lambda_{2}}$} & \multicolumn{3}{c}{$e_{x}$} \\
\hline & Mean & Min & Max & Mean & Min & Max \\
\hline 10 & $2.64 e-12$ & $1.29 e-16$ & $2.31 e-09$ & $5.78 e-14$ & $2.83 e-17$ & $1.82 e-14$ \\
25 & $1.38 e-14$ & $1.51 e-16$ & $2.72 e-12$ & $6.83 e-16$ & $1.98 e-17$ & $4.84 e-14$ \\
50 & $2.90 e-14$ & $2.06 e-16$ & $2.28 e-11$ & $7.46 e-16$ & $1.36 e-17$ & $7.98 e-14$ \\
75 & $3.46 e-13$ & $3.71 e-16$ & $3.42 e-10$ & $1.08 e-14$ & $1.28 e-17$ & $1.01 e-11$ \\
100 & $7.15 e-15$ & $3.40 e-16$ & $3.21 e-12$ & $6.90 e-16$ & $9.96 e-18$ & $8.27 e-14$
\end{tabular}

On the other hand, the CPU time of Algorithms 1 and 2 are measured in seconds, using the Matlab TimeCPU routine. The first column of Table 9 displays the dimension $n$. To display the CPU times there are three columns for both algorithms: the first one displays the average over the 1000 tests, while the second and third columns display, respectively, the smallest and largest value for the CPU time among the 1000 experiments.

Table 9: The CPU time of Algorithms 1 and 2

\begin{tabular}{ccccccc}
\hline$n$ & \multicolumn{2}{c}{ CPU Time Alg 1 } & \multicolumn{3}{c}{ CPU Time Alg 2} \\
\hline & Mean & Min & Max & Mean & Min & Max \\
\hline 10 & $8.61 \mathrm{e}-14$ & $7.78 \mathrm{e}-04$ & $7.91 \mathrm{e}-03$ & $9.82 \mathrm{e}-04$ & $8.95 \mathrm{e}-04$ & $1.61 \mathrm{e}-03$ \\
25 & $4.79 \mathrm{e}-03$ & $4.37 \mathrm{e}-03$ & $1.57 \mathrm{e}-02$ & $6.25 \mathrm{e}-03$ & $4.95 \mathrm{e}-03$ & $1.71 \mathrm{e}-02$ \\
50 & $2.09 \mathrm{e}-02$ & $1.96 \mathrm{e}-02$ & $3.32 \mathrm{e}-02$ & $2.59 \mathrm{e}-03$ & $2.16 \mathrm{e}-02$ & $8.67 \mathrm{e}-02$ \\
75 & $5.42 \mathrm{e}-02$ & $5.10 \mathrm{e}-02$ & $8.59 \mathrm{e}-02$ & $6.25 \mathrm{e}-02$ & $5.44 \mathrm{e}-02$ & $1.37 \mathrm{e}-01$ \\
100 & $1.11 \mathrm{e}-01$ & $1.31 \mathrm{e}-01$ & $1.31 \mathrm{e}-01$ & $1.19 \mathrm{e}-01$ & $1.08 \mathrm{e}-01$ & $1.36 \mathrm{e}-01$
\end{tabular}

As we can see in the tables, both algorithms find solutions whose spectral data are approximately close to the initial data and are consistently fast.

Data Availability Statement No data were used to support this study. 


\section{Acknowledgements.}

The research of H. Pickmann was supported by Universidad de Tarapacá, Arica, Chile. The research of S. Arela was supported by Universidad de Tarapacá, Arica, Chile. The research of J. Egaña was supported by Universidad Católica del Norte, Antofagasta, Chile. The research of D. Carrasco was partially supported by FONDECYT project 1181061, Agencia Nacional de Investigación y Desarrollo-ANID, Chile and by project 196108 GI/C, Grupo de Investigación en Sistemas Dinámicos y AplicacionesGISDA, UBB, Chile.

\section{References}

[1] D. Boley and G. H. Golub, "A survey of matrix inverse eigenvalue problems", Inverse problems, vol. 3, no. 4, pp. 595-622, 1987, doi: $10.1088 / 0266-5611 / 3 / 4 / 010$

[2] M. T. Chu and G. H. Golub, Inverse eigenvalue problems: theory, algorithms, and applications. Oxford: Oxford University Press, 2005, doi: 10.1093/acprof:oso/ 9780198566649.001 .0001

[3] G. M. Gladwell, Inverse problems in vibratio, Dordrecht: Springer, 1986.

[4] V. Higgins and C. Johnson, "Inverse spectral problems for collections of leading principal submatrices of tridiagonal matrices", Linear algebra and its applications, vol. 489, pp. 104-122, Jan. 2016, doi: 10.1016/ j.laa.2015.10.004

[5] H. Hochstadt, "On the construction of a Jacobi matrix from mixed given data", Linear algebra and its applications, vol. 28, pp. 113-115, Dec. 1979, doi: 10.1016/0024-3795(79)90124-1

[6] D. Inman, Vibration with control, measurement and stability. Englewood Ciffs, NJ: Prentice-Hall, 1989.

[7] N. Jakovčevic Stor, I. Slapničar, and J. Barlow, "Accurate eigenvalue decomposition of real symmetric arrowhead matrices and applications", Linear algebra and its applications, vol. 464, pp. 62-89, Jan. 2015, doi: 10.1016/ j.laa.2013.10.007

[8] A. M. Nazari and Z. Beiranvand, "The inverse eigenvalue problem for symmetric quasi anti-bidiagonal matrices", Applied mathematics and computation, vol. 217, no. 23, pp. 9526-9531, Aug. 2011, doi: 10.1016/ j.amc.2011.03.031 
[9] J. Peng, X. Y. Hu, and L. Zhang, "Two inverse eigenvalue problems for a special kind of matrices", Linear algebra and its applications, vol. 416, no. 2-3, pp. 336-347, Jul. 2006, doi: 10.1016/ j.laa.2005.11.017

[10] H. Pickmann, J. Egaña, and R. L. Soto. "Extremal inverse eigenvalue problem for bordered diagonal matrices", Linear algebra and its applications, vol. 427, no. 2-3, pp. 256-271, Dec. 2007, doi: $10.1016 /$ j.laa.2007.07.020

[11] H. Pickmann, J. Egaña, and R. L. Soto, "Two inverse eigenproblems for symmetric doubly arrow matrices", Electronic journal of linear algebra, vol. 18, pp. 700-718, Jan. 2009, doi: 10.13001/ 1081-3810.1339

[12] H. Pickmann, J. Egaña, and R. L. Soto, "Extreme spectra realization by real symmetric tridiagonal and real symmetric arrow matrices", Electronic journal of linear algebra, vol. 22, pp. 780-795, Jan. 2011, doi: 10.13001/1081-3810.1474

[13] H. Pickmann, S. Arela, J. Egaña, and D. Carrasco, "On the inverse eigenproblem for symmetric and nonsymmetric arrowhead matrices", Proyecciones (Antofagasta, On line), vol. 38, no. 4, pp. 811-828, Oct. 2019, doi: 10.22199/ issn.0717-6279-2019-04-0053 